\title{
NanoVelcro-captured CTC number concomitant with enhanced serum levels of MMP7 and MMP9 enables accurate prediction of metastasis and poor prognosis in patients with lung adenocarcinoma
}

\author{
This article was published in the following Dove Press journal: \\ International Journal of Nanomedicine \\ 31 August 2017 \\ Number of times this article has been viewed
}

\author{
Yu Sun ${ }^{1, *}$ \\ Yangshan Chen ${ }^{1, *}$ \\ Shuhua $\mathrm{Li}^{1}$,* \\ Yiyan Lei ${ }^{2}$ \\ Di Xu ${ }^{3}$ \\ Neng Jiang' \\ Yang Zhang ${ }^{4}$ \\ Jessica $\mathrm{CaO}^{5}$ \\ Zunfu Ke ${ }^{1, *}$ \\ 'Department of Pathology, \\ 2Department of Thoracic Surgery, \\ The First Affiliated Hospital, Sun \\ Yat-sen University, Guangzhou, \\ Guangdong, People's Republic of \\ China; ${ }^{3}$ Department of Thoracic \\ Surgery, The Central Hospital of \\ Wuhan, Wuhan, Hubei, People's \\ Republic of China; ${ }^{4}$ Biomedical \\ Engineering, The University of \\ Texas at El Paso, El Paso, TX, USA; \\ ${ }^{5}$ Health Sciences, Cumming School \\ of Medicine, University of Calgary, \\ Calgary, Alberta, Canada \\ *These authors contributed equally \\ to this work
}

\begin{abstract}
Lung adenocarcinoma (LADC) is among the most malignant cancers that frequently develops micrometastases even in early stages of the disease. Circulating tumor cell (CTC) number, matrix metalloproteinase (MMP) 7, and MMP9 show great prospects as predictive biomarkers in many tumors. However, the interactions between these biomarkers and the molecular basis of their roles in the metastasis and prognosis of LADC remain unclear. The present study revealed that an elevated CTC count and overexpression of MMP7 and MMP9 correlate with metastasis and clinical progression in LADC patients $(n=143)$. Furthermore, MMP7 and MMP9 upregulation facilitates LADC cell migration in vitro and enhances serum CTC levels in a xenograft mouse model. More importantly, receiver operating characteristic (ROC) curves and Kaplan-Meier analysis confirmed more accurate prediction of metastasis and overall survival (OS) with a combination panel of CTC, MMP7, and MMP9. Taken together, our data show, for the first time, the involvement of MMP7 and MMP9 in the release of CTCs into the peripheral blood, and our data reveal that CTC count and expression of MMP7 and MMP9 can be used together as an effective clinical prediction panel for LADC metastasis and prognosis.

Keywords: lung adenocarcinoma, circulating tumor cell, MMP7/MMP9, metastasis, prognosis
\end{abstract}

\section{Introduction}

Lung cancer is the foremost cause of cancer-related mortality worldwide, leading to approximately 1.6 million deaths every year, mostly attributable to the tobacco epidemic and widespread air pollution. ${ }^{1,2}$ Lung adenocarcinoma (LADC), the most common and invasive histological subtype of non-small-cell lung cancer (NSCLC), accounts for more than $40 \%$ of lung cancers. ${ }^{3}$ Despite recent advances in surgery, radiotherapy, chemotherapy, and molecularly targeted treatments, the cure rate remains discouraging as many patients have already developed micrometastases during the earliest stages. ${ }^{4}$ A substantial percentage of patients present with inoperable, regional, or distant disseminated disease by the time they are diagnosed, rendering curative treatment even more difficult..$^{5}$ Thus, there remains an urgent need to identify more sensitive and specific biomarkers to precisely predict the prognosis of patients with inchoate LADC and potentially allow for early intervention.

In order for metastasis to occur, circulating tumor cells (CTCs) must detach from the primary tumor, migrate into the bloodstream, and spread to distant tissues at different stages of malignant progression. The isolation and analysis of CTCs are also known 
as a "liquid biopsy," and this technique provides convenient and noninvasive access to both the primary tumor and distant metastases. ${ }^{6}$ Recently, evidence has demonstrated that CTCs may be used to determine prognosis in breast, colorectal, and prostate cancers. ${ }^{7-9}$ Some studies have further demonstrated that CTCs can be used as a surrogate biomarker in SCLC patients receiving targeted therapy. ${ }^{10}$ However, the molecular mechanisms governing the detachment of CTCs from primary tumor sites to eventually form lethal metastases in LADC remain unclear.

Changes in the extracellular matrix (ECM), which include the loss of intercellular adhesion molecules and the expression of proteolytic enzymes, have been proposed to be a mechanistic driver for CTC formation. ${ }^{11}$ Emerging studies suggested that matrix metalloproteinases (MMPs), a family of highly conserved proteolytic enzymes, possess the ability to degrade the ECM, weaken cell-matrix interaction, and facilitate the dissemination of tumor cells. ${ }^{12-16}$ A previous study found that MMP7 is upregulated by cyclooxygenase-2 (COX-2) to promote tumor growth and lymph node metastasis. ${ }^{17}$ Additionally, Cox et al ${ }^{18}$ suggested that MMP9 may play a crucial role in the invasive behavior of NSCLC. Our previous research demonstrated that MMP9 can be regulated by AEG-1 to promote progression in NSCLC, consistent with published findings. ${ }^{19}$ Overall, MMP7 and MMP9, as well as CTCs, have repeatedly been implicated in the aggressiveness of malignant solid tumors. However, little is known about the roles of these potential biomarkers in LADC, and even less is known about the interaction between CTC number and the serum levels of MMP7/9.

In the present study, our results show that an elevated CTC count, concomitant with enhanced serum levels of MMP7 and MMP9, is associated with metastasis and poor OS in LADC patients. We further uncover a mechanism by which MMP7 and MMP9 can promote CTC detachment by degrading the ECM, thus highlighting the utility of a novel panel composed of CTC counts, serum MMP7 levels, and serum MMP9 levels that is capable of predicting LADC metastasis and prognosis with greater accuracy.

\section{Materials and methods}

\section{Patients}

A total of 143 participants diagnosed with LADC were included in this study. Written informed consent was obtained from every participant. Patients' corresponding clinical characteristics were obtained from the archives of the First Affiliated Hospital of Sun Yat-sen University and the Central Hospital of Wuhan. Peripheral blood samples were collected into ethylenediaminetetraacetic acid (EDTA)-containing vacutainer tubes (BD Bioscience, San Jose, CA, USA) from all patients before the initiation of standard treatment for use in further procedures. This study was approved by the institutional ethics committee of the First Affiliated Hospital of Sun Yat-sen University.

\section{CTC enrichment by the NanoVelcro system}

CTC enrichment was performed using the NanoVelcro system as described in our previous paper. ${ }^{20}$ Blood samples ( $1 \mathrm{~mL}$ of venous blood) were collected in EDTA tubes and processed within $24 \mathrm{~h}$. Then, $\mathrm{NH}_{4} \mathrm{Cl}$ was added to whole blood in the ratio of $10: 1 \mathrm{v} / \mathrm{v}$, followed by a $20 \mathrm{~min}$ incubation at room temperature until the red blood cells lysed. After centrifugation at $200 \mathrm{~g}$ for $5 \mathrm{~min}$, the sample supernatant was removed, and the cell pellets were resuspended. Subsequently, immunocytochemistry was used to identify and quantify the CTCs captured on the Si nanowire array (SiNW) substrate.

\section{Immunofluorescence staining of captured CTCs}

Captured CTCs were fixed with phosphate buffered solution (PBS) containing 2.0\% formaldehyde, washed, and blocked with $1 \%$ donkey sera in PBS. Then, a commonly used three-color immunocytochemistry method was utilized to discriminate CTCs from white blood cells (WBCs), including TRITC-conjugated anti-CD45 (CD45, a marker for WBCs), FITC-conjugated anticytokeratin (CK, a protein marker for epithelial cells), as well as 4,6-diamino-2-phenyl indole (DAPI) nuclear staining. As shown in Figure 1B, CTCs exhibit CK+/CD45-/DAPI+, while WBCs express CK-/CD45+/DAPI+.

\section{Enzyme-linked immunosorbent assay (ELISA)}

Sera were obtained from all LADC patients. Serum concentrations of MMP7 and MMP9 were quantified by ELISA using the MMP7, MMP9 ELISA kit (Boster, People's Republic of China), respectively. ELISA was performed following the manufacturer's instructions. Protein concentrations were determined by comparing the relative absorbance of the samples with known standards. All samples were examined in triplicate, and the mean values were used in subsequent statistical analyses.

\section{Cell lines and culture conditions}

LADC cell lines NCI-H1975 and NCI-H2122 were purchased from the American Type Culture Collection (ATCC). 
A
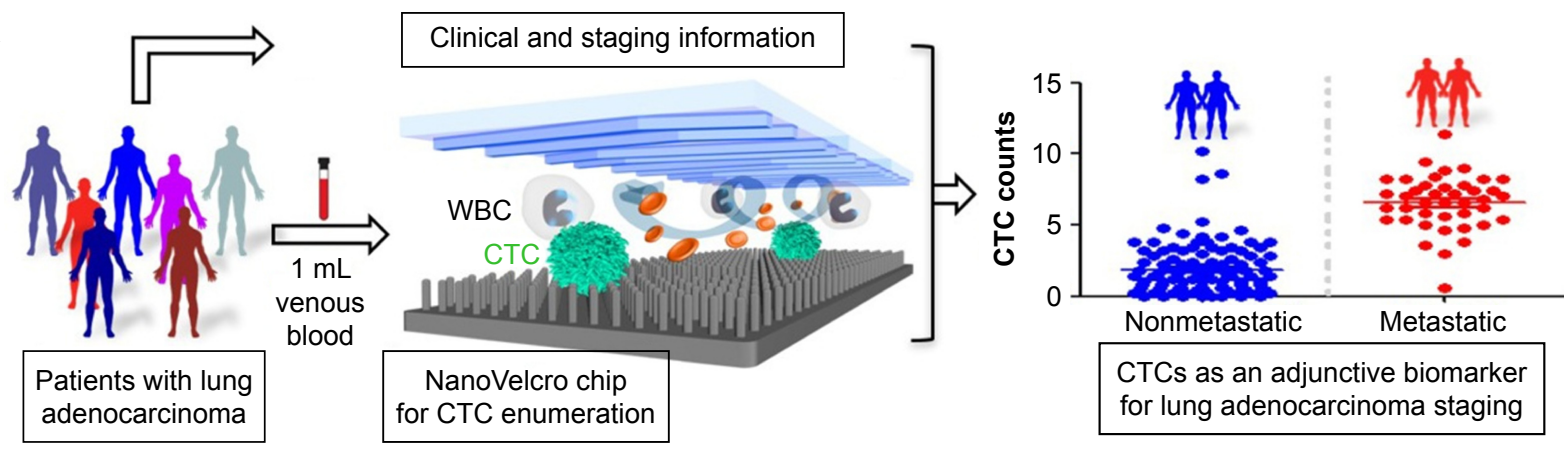

B
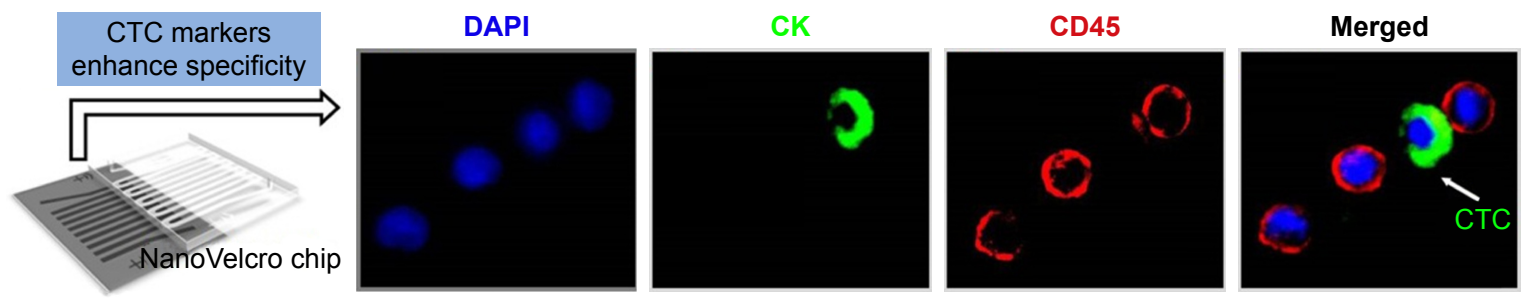

Figure I CTC detection and verification in LADC.

Notes: (A) Whole blood was obtained from 143 patients with LADC and then processed using the NanoVelcro system. The CTC count was significantly elevated in patients with metastasis. (B) CTCs were identified by the colocalization of DAPI (blue) and CK (green) fluorescence, while WBCs were identified by CD45 (red) and DAPI (blue) fluorescence. Abbreviations: CTC, circulating tumor cell; DAPI, 4,6-diamino-2-phenyl indole; LADC, lung adenocarcinoma; WBC, white blood cell.

Cells were maintained in Dulbecco's Modified Eagle's Medium (DMEM; Invitrogen, Carlsbad, CA, USA), supplemented with 10\% foetal bovine serum (HyClone, San Angelo, TX, USA).

\section{Plasmids, retroviral infection, and transfection}

The MMP7 and MMP9 constructs were generated by subcloning polymerase chain reaction (PCR)-amplified fulllength human MMP7 or MMP9 cDNA into pcDNA3.1. For depletion of MMP7 and MMP9, siRNA sequences were cloned into GV248 to generate GV248-RNAi(s) targeting MMP7 and MMP9. siRNA duplexes were synthesized and purified by RiboBio Inc (Guangzhou, People's Republic of China). The siRNA sequences are listed in Table S1. The PCR primers are listed in Table S2. siRNA transfection was carried out using Lipofectamine 2000 reagent (Invitrogen). Retroviral production and infection were performed according to the manufacturer's instructions. Following selection, lung cancer cell lysates prepared from the pooled cell populations in sampling buffer were fractionated by sodium dodecyl sulfate-polyacrylamide gel electrophoresis (SDS-PAGE) to detect protein levels via Western blotting.

\section{Western blotting}

Western blotting was performed as previously described. ${ }^{21}$ After incubation with primary antibodies in 5\% milk/TBST at $4{ }^{\circ} \mathrm{C}$ overnight, the membranes were washed with TBST. Then, the membranes were incubated for $60 \mathrm{~min}$ with HRP-conjugated antirabbit immunoglobulin G ( $\mathrm{IgG}$ ) at room temperature. The membranes were washed with TBST, and signals were detected via enhanced chemiluminescence (ECL).

\section{Adhesion assay}

The adhesive capacities of NCI-H1975 and NCI-H2122 cells were tested by cell adhesion experiments. Ninety-six-well plates were coated with fibronectin $(10 \mu \mathrm{g} / \mathrm{mL})$ in PBS for $2 \mathrm{~h}$ at $37^{\circ} \mathrm{C}$ and then blocked with $1 \%$ bovine serum albumin (BSA). After centrifugation and suspension (using nonserum RPMI 1640 medium containing $0.1 \%$ BSA and $0.1 \%$ glucose), cells were collected. In triplicate, $75 \times 10^{4}$ cells $/ \mathrm{mL}$ in a volume of $100 \mu \mathrm{L}$ were plated and incubated for the indicated time periods $(24 \mathrm{~h}, 48 \mathrm{~h}$, and $72 \mathrm{~h})$ at $37^{\circ} \mathrm{C}$. Then, the plates were carefully washed with PBS to remove nonadherent cells. The presence of adherent cells was confirmed using an IX71 inverted microscope (Olympus Corp, Tokyo, Japan).

\section{Transwell assay}

Cell migration assays were performed in triplicate using $8-\mu \mathrm{m}$ pore filters with $6.5-\mathrm{mm}$-diameter chambers in 24-well plates (Costar, Boston, MA, USA). Then, $50 \mu \mathrm{L}$ of fibronectin $(100 \mu \mathrm{g} / \mathrm{mL})$ was coated on the lower filter surfaces. Subsequently, the filters were air-dried. ${ }^{22}$ NCI-H1975 
and NCI-H2122 cells were suspended at $2 \times 10^{5}$ cells $/ \mathrm{mL}$ in serum-free media and loaded in the upper chambers at a volume of $200 \mu \mathrm{L}$. Serum-free medium was added in the lower chambers, followed by incubation in a humidified atmosphere containing $5 \% \mathrm{CO}_{2}$ for $12 \mathrm{~h}$ at $37^{\circ} \mathrm{C}$. After incubation, the filters were fixed in $4 \%$ paraformaldehyde for $10 \mathrm{~min}$ at room temperature, and HE staining was performed. Nonmigrated cells on the upper filter surfaces were removed with cotton swabs. The numbers of migrated cells were quantified in five high-power $(400 \times)$ fields per filter under a microscope (Olympus Corp).

\section{Xenograft tumor model}

Six-week-old male NOD/SCID mice were purchased from SLAC (Shanghai, People's Republic of China). All animal experiments were conducted in accordance with the Guide for the Care and Use of Laboratory Animals and approved by the Institutional Animal Care and Use Committee of Sun Yat-sen University. After subcutaneous injection of $2 \times 10^{6}$ different versions of NCI-H1975 cells and control cells (in $100 \mu \mathrm{L}$ of PBS) into the forearm of each mouse $(n=8)$, the mice were examined 3 times per week for evidence of morbidity and mortality. On days 10, 20, and 30 after inoculation, $0.1 \mathrm{~mL}$ of blood was obtained from the forearm of each mouse. Bioluminescence imaging of the tumors was conducted on day 30 using an IVIS system, followed by euthanization of the mice via cervical dislocation.

\section{Statistical analysis}

The data are presented as the mean \pm standard deviation (SD). SPSS 16.0 (SPSS Inc., Chicago, IL, USA) was used for statistical analyses. Scatter diagrams and bar charts were plotted using GraphPad Prism 5.0. A $p$-value of $<0.05$ was considered statistically significant. Unpaired, two-tailed Student's $t$-test was used to determine the differences between groups. The Spearman test was used to compute the concordance rate between pathology tumor-node-metastasis (pTNM) stage and CTC count/serum MMP7 expression/serum MMP9 expression. The concordance rate of linear regression plots was assessed using the Pearson test. To determine the optimal cutoffs of CTC count, serum MMP7 expression and serum MMP9 expression for evaluating metastasis, we performed a nonparametric ROC curve analysis. Univariate OS was calculated using Kaplan-Meier curves and compared with the log-rank test. The combined analysis of CTC count, MMP7 expression, and MMP9 expression was predicted by logistic regression analysis, and the exported statistics were used to plot the ROC curve and survival curve.

\section{Results \\ Association of CTC number with LADC metastasis status}

To investigate the effect of CTCs on the metastasis status, we first applied NanoVelcro to quantify CTCs in the blood samples of 143 LADC patients with different pTNM stages (Figure 1A). Immobilized CTCs were further confirmed by immunofluorescence to be CD45-negative and CK-positive, as is typical (Figure 1B). Statistical results revealed that the CTC count was significantly higher in LADC patients with metastasis (mean values: $6.6 \pm 1.92 \mathrm{CTC} \mathrm{mL}^{-1}$ ) than in those without metastasis (mean values: $1.9 \pm 1.85 \mathrm{CTC} \mathrm{mL}^{-1}$ ) (Figure 2B). Furthermore, we found that the CTC count was correlated with pTNM stage ( $p<0.01$; Figure 2 C). Additionally, CTC formation was reported to be facilitated by larger tumor size in many solid tumors. ${ }^{23-27}$ However, there was no significant correlation between tumor size and CTC count in our results (Figure 2D; Table 1).

\section{Correlation of CTC number, MMP7 expression, and MMP9 expression with LADC progression}

As members of the MMP family of zinc-dependent endopeptidases, MMP7 and MMP9 possess the ability to degrade ECM, which is thought to play an indispensable role in cancer invasion and metastasis. ${ }^{17,18}$ To determine whether MMP7 and MMP9 are linked to the malignant progression of LADC and the detachment of CTCs in LADC, we detected their expression by ELISA. We observed that patients with metastasis had significantly higher expression levels of MMP7 and MMP9 compared to those without metastasis (Figure 3A). Further analysis revealed that advanced tumor stages were associated with much higher serum MMP7 and MMP9 levels ( $p<0.01$; Figure 3B and C). In addition, linear regression plots showed a strong positive correlation between CTC count and the serum levels of MMP7 and MMP9 $(p<0.01$; Figure $3 \mathrm{D}$ and E). Overall, these data suggest that CTC count might be influenced by MMP7 and MMP9 expression levels in patients with LADC. The presence of CTCs, MMP7, and MMP9 indicates a higher probability of metastasis and worse clinical outcomes.

\section{MMP7 and MMP9 promote the migration of LADC cells in vitro and in vivo}

Based on the foregoing data, we hypothesized that MMP7 and MMP9 may facilitate CTC detachment by weakening the cell-matrix interaction. To provide direct evidence for this assumption, we used the NCl-H1975 and NCl-H2122 

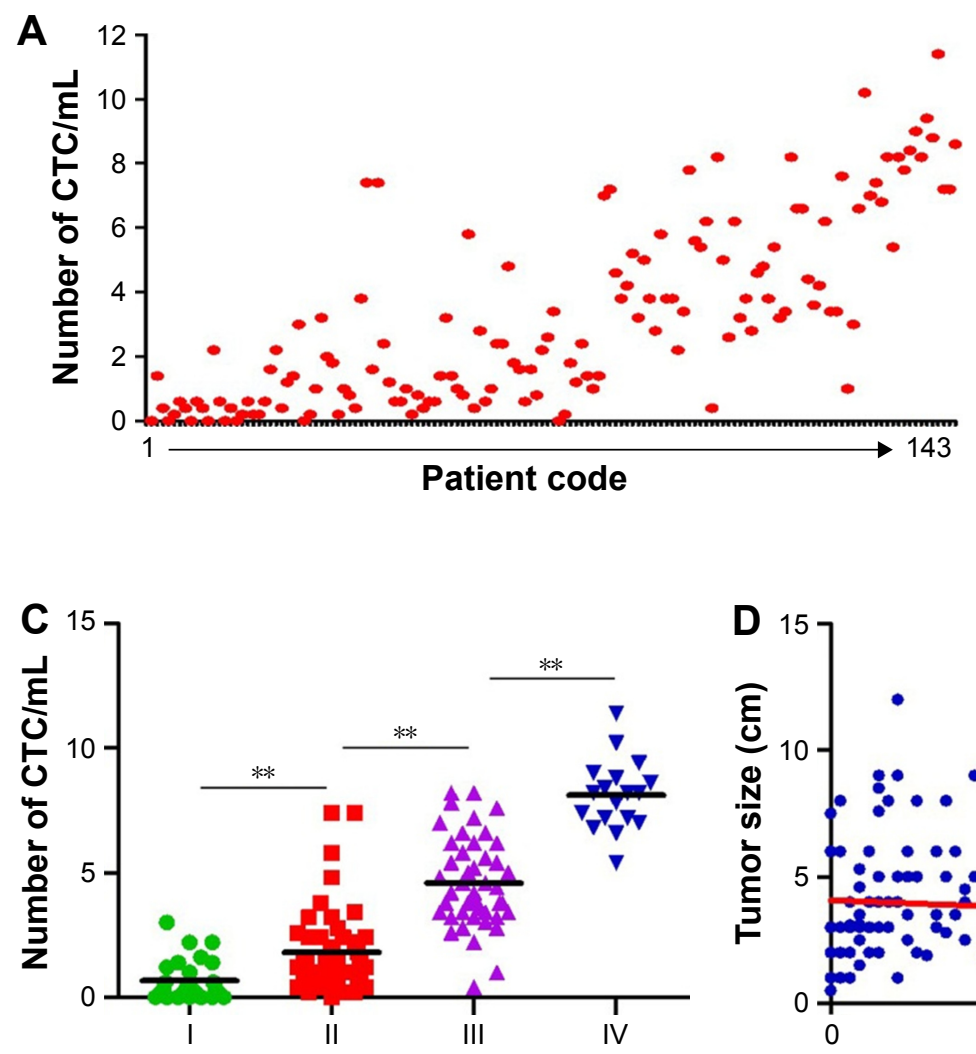

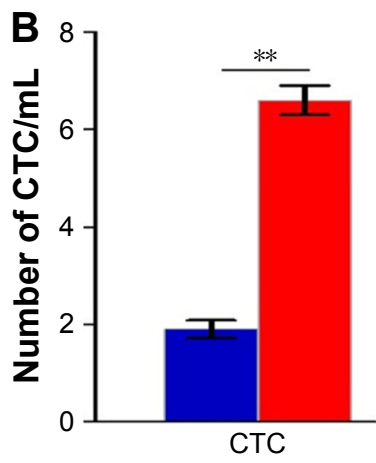

Without metastasis With metastasis

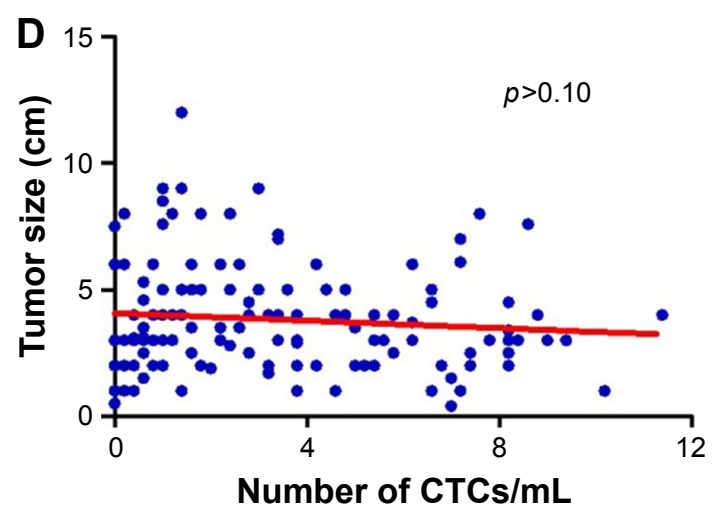

Figure 2 Elevated CTC count in LADC correlates with poor clinical prognosis.

Notes: (A) The distribution of CTC counts in the peripheral blood samples of 143 LADC patients based on NanoVelcro. (B) CTC number was significantly increased in patients with metastasis compared with those without metastasis $(* * p<0.0 \mathrm{I})$. (C) Patients with increased CTC numbers were at advanced $\mathrm{pTNM}$ stages $(* * p<0.0 \mathrm{I})$. (D) CTC count and tumor size were not significantly correlated $(p>0.10)$

Abbreviations: CTC, circulating tumor cell; LADC, lung adenocarcinoma; PTNM, pathology tumor-node-metastasis.

Table I Correlation between CTC counts and clinicopathologic characteristics of LADC

\begin{tabular}{|c|c|c|c|c|c|c|c|c|c|}
\hline \multirow[t]{2}{*}{$\begin{array}{l}\text { Clinicopathologic } \\
\text { characteristics }\end{array}$} & \multicolumn{2}{|c|}{ CTC counts } & \multirow[t]{2}{*}{$p$-value } & \multicolumn{2}{|c|}{$\begin{array}{l}\text { MMP7 } \\
\text { expression }\end{array}$} & \multirow[t]{2}{*}{$p$-value } & \multicolumn{2}{|c|}{$\begin{array}{l}\text { MMP9 } \\
\text { expression }\end{array}$} & \multirow[t]{2}{*}{$\overline{p \text {-value }}$} \\
\hline & High & Low & & High & Low & & High & Low & \\
\hline Tumor size $(\mathrm{cm})$ & & & 0.062 & & & 0.075 & & & 0.103 \\
\hline$\leq 3 \mathrm{~cm}$ & 23 & 47 & & 28 & 42 & & 25 & 45 & \\
\hline$>3 \mathrm{~cm}$ & 14 & 59 & & 19 & 54 & & 17 & 56 & \\
\hline Age (years) & & & 0.004 & & & 0.286 & & & 0.906 \\
\hline$\leq 50$ & 26 & 45 & & 26 & 44 & & 20 & 47 & \\
\hline$>50$ & II & 61 & & 21 & 52 & & 22 & 54 & \\
\hline Gender & & & 0.724 & & & $0.4 \mathrm{II}$ & & & 0.405 \\
\hline Male & 18 & 48 & & 23 & 40 & & 18 & 51 & \\
\hline Female & 19 & 58 & & 24 & 56 & & 24 & 50 & \\
\hline Smoking & & & $<0.001$ & & & 0.235 & & & 0.627 \\
\hline Yes & 27 & 35 & & 20 & 51 & & 21 & 55 & \\
\hline No & 10 & 7I & & 27 & 45 & & 21 & 46 & \\
\hline Differentiation & & & 0.922 & & & 0.370 & & & 0.062 \\
\hline Well & 8 & 26 & & 14 & 33 & & 9 & 41 & \\
\hline Moderately & 14 & 37 & & 17 & 41 & & 14 & 31 & \\
\hline Poorly & 15 & 43 & & 16 & 22 & & 19 & 29 & \\
\hline Clinical stage & & & $<0.001$ & & & $<0.001$ & & & $<0.001$ \\
\hline I & 0 & 30 & & 0 & 30 & & 0 & 30 & \\
\hline II & 3 & 47 & & 4 & 46 & & 3 & 47 & \\
\hline III & 16 & 29 & & 26 & 19 & & 21 & 24 & \\
\hline IV & 18 & 0 & & 17 & I & & 18 & 0 & \\
\hline
\end{tabular}

Note: Statistical significance $(p<0.05)$ is shown in bold.

Abbreviations: CTC, circulating tumor cell; MMP7/9, matrix metalloproteinase 7/9. 
A

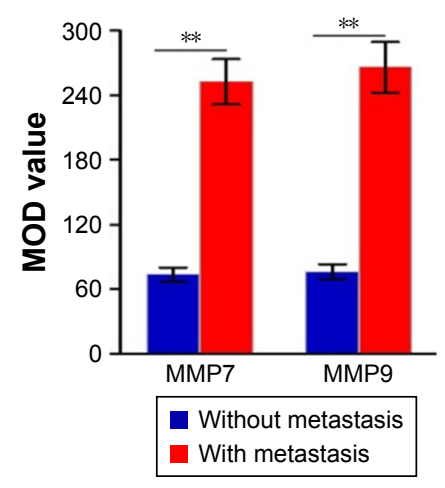

D

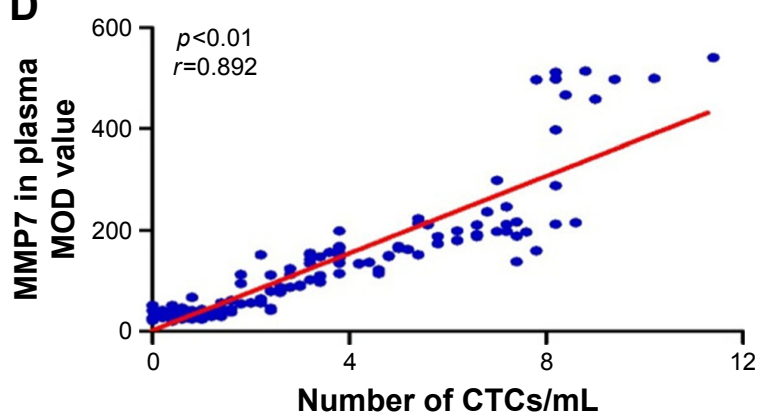

B

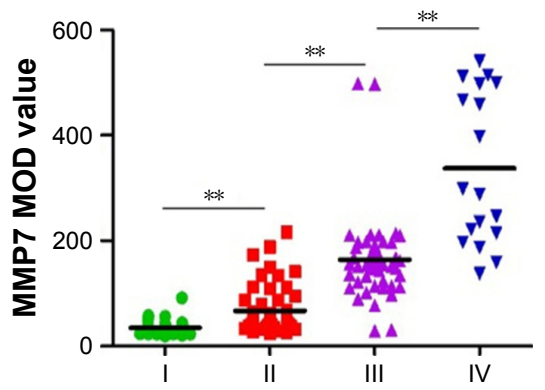

C

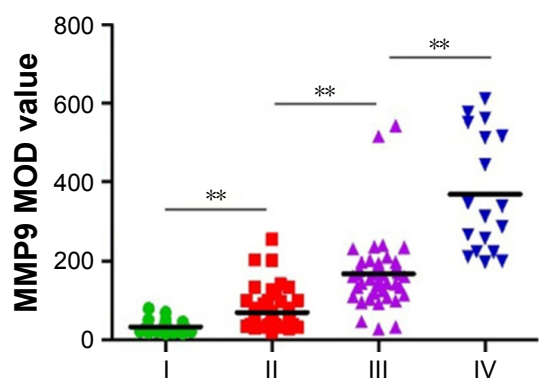

E

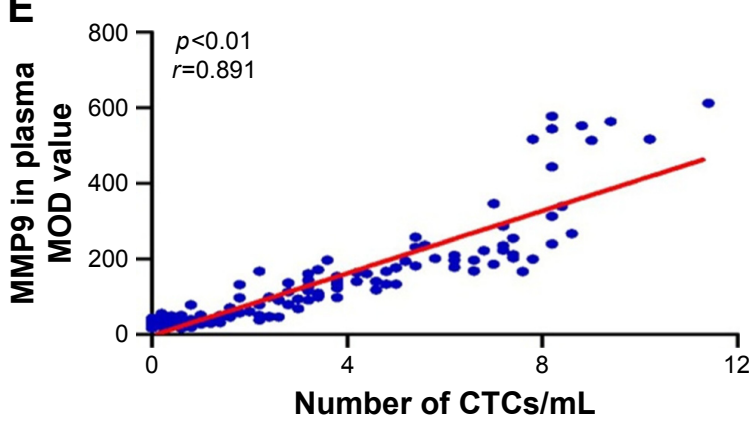

Figure 3 MMP7 and MMP9 correlate with CTC count and poor clinical prognosis.

Notes: (A) Serum levels of MMP7 and MMP9 were significantly increased in LADC patients with metastasis compared to those without metastasis (** $<<0.01$ ). (B, C) Remarkable associations were observed between PTNM stage and MMP7 and MMP9 expression levels (**p<0.0I). (D, E) CTC counts were positively correlated with serum MMP7 $(r=0.892 ; p<0.01)$ and MMP9 $(r=0.89$ I; $p<0.01)$ expression levels.

Abbreviations: CTC, circulating tumor cell; MMP7/9, matrix metalloproteinase 7/9; MOD, mean optical density.

cell lines to establish cell lines in which MMP7/9 were stably overexpressed or depleted. The overexpression and knockdown of MMP7/9 were further confirmed by Western blotting (Figure 4A). An adhesion assay revealed that decreased tumor cell adhesion was accompanied by the ectopic overexpression of MMP7/9, while depletion of MMP7/9 increased tumor cell adhesion. Of note, the number of adherent LADC cells was predominantly decreased when MMP7 and MMP9 were upregulated together, whereas the adhesion index was significantly elevated on depletion of both MMP7 and MMP9
(Figure 4B). Furthermore, compared to overexpression of MMP7 and MMP9 individually, the overexpression of both MMP7 and MMP9 greatly increased the capacity of tumor cells to invade through a Transwell gel, and depletion of both MMP7 and MMP9 suppressed tumor cell invasion $(p<0.01$; Figure 4C and D).

We further established a xenograft mouse model, using the cell line NCI-H1975, in which MMP7/9 were stably overexpressed or knocked down separately or simultaneously (Figure 5A). Different versions of cell lines and control
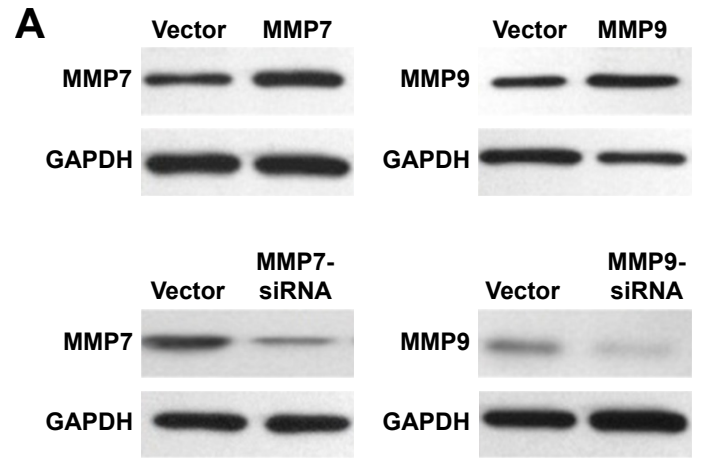
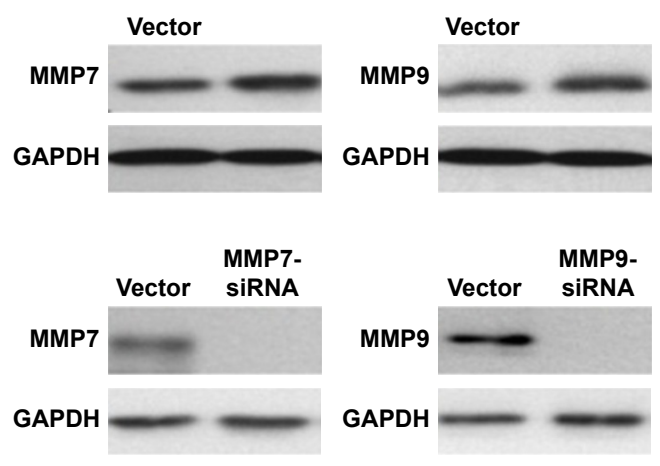

Figure 4 (Continued) 

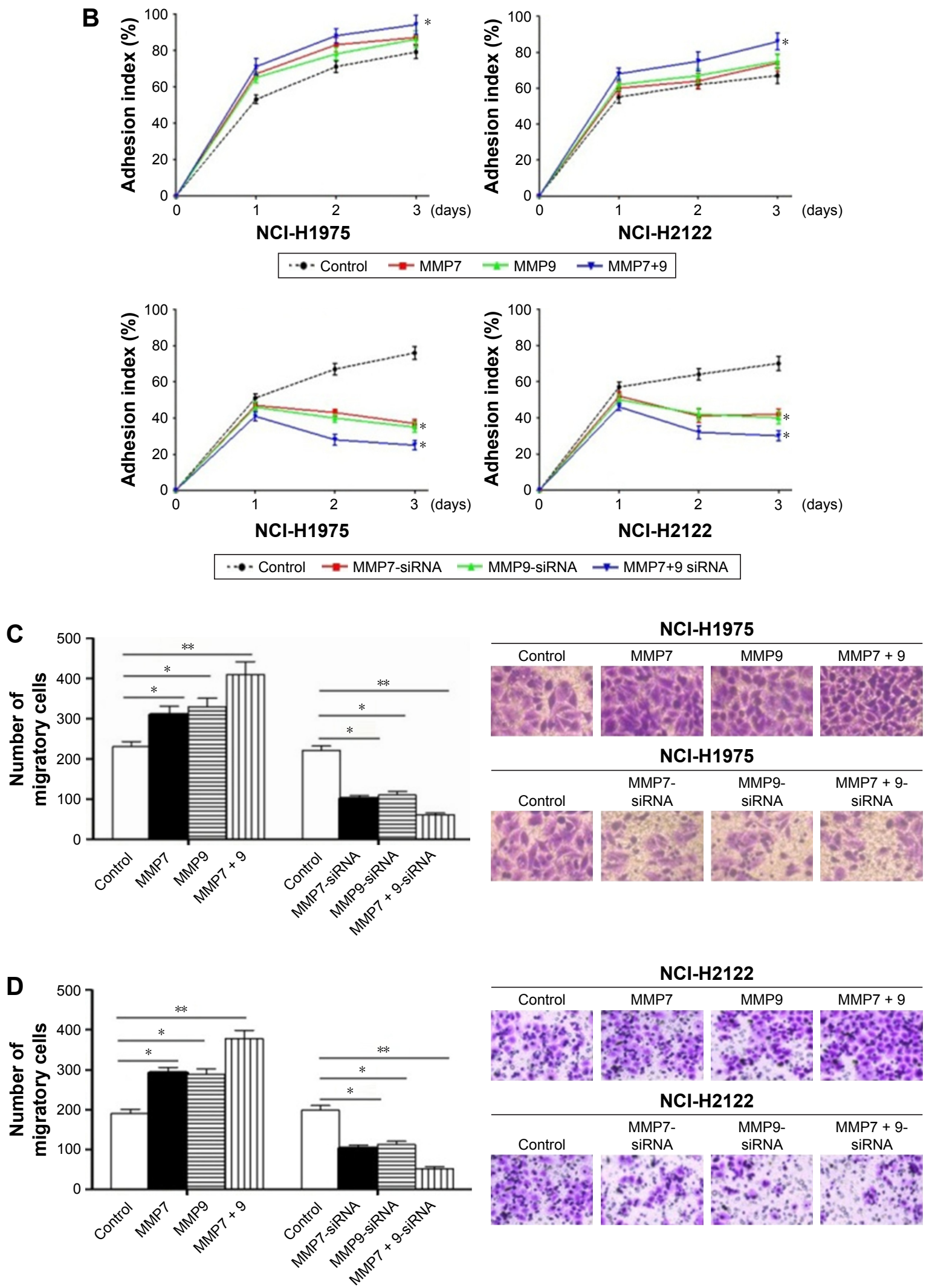

Figure 4 MMP7 and MMP9 inhibit adhesion and promote LADC cell migration and invasion in vitro.

Notes: (A) Stable overexpression or knockdown of endogenous MMP7/MMP9 in LADC cell lines (NCl-HI975 and NCl-H2I22) was confirmed by Western blotting. (B) MMP7 and MMP9 overexpression inhibited cell adhesion; MMP7 and MMP9 depletion increased cell adhesion. (C, D) Ectopic overexpression of MMP7 and MMP9 promoted cell invasion $(* * p<0.01)$; knockdown of endogenous MMP7 and MMP9 inhibited cell invasion $(* * p<0.01)$. $*^{*}<0.05$.

Abbreviations: LADC, lung adenocarcinoma; MMP7/9, matrix metalloproteinase 7/9. 
A

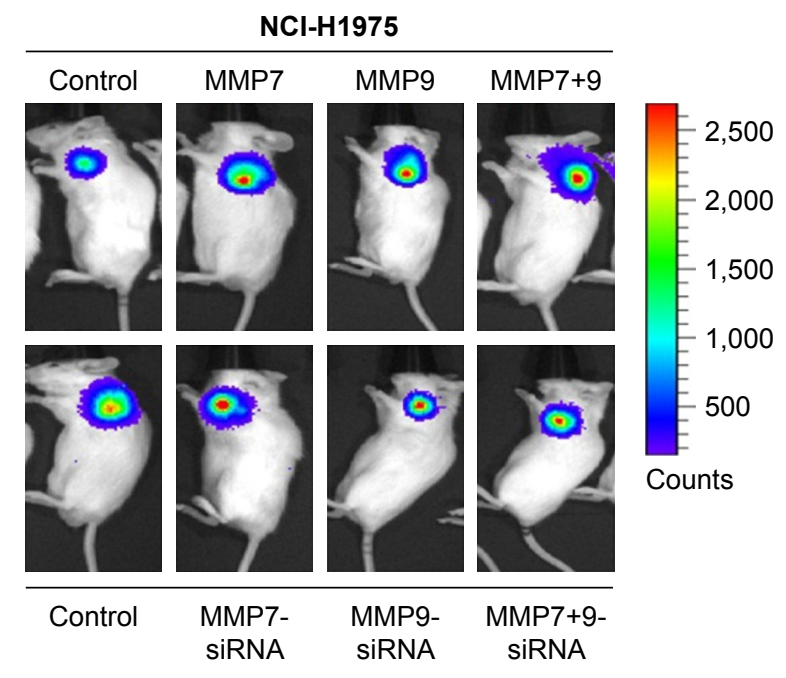

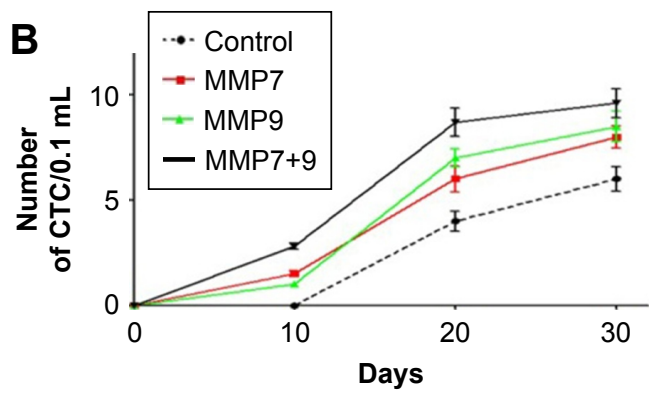

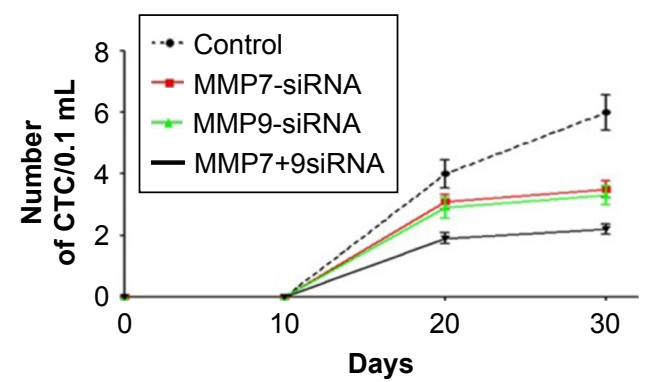

Figure 5 MMP7 and MMP9 facilitate the liberation of LADC cells into the bloodstream in vivo.

Notes: (A) Bioluminescence imaging of NOD/SCID mice 30 days after subcutaneous injection of different versions of NCl-HI975 cells and control cells. (B) MMP7 and MMP9 overexpression decreased CTC levels; MMP7 and MMP9 depletion increased CTC count.

Abbreviations: CTC, circulating tumor cell; LADC, lung adenocarcinoma; MMP7/9, matrix metalloproteinase 7/9.

cells were subcutaneously injected into NOD/SCID mice $(\mathrm{n}=8)$. CTC counts in $0.1 \mathrm{~mL}$ of blood that were quantified by NanoVelcro on days $0,10,20$, and 30 are presented in Figure 5B. Consistent with our in vitro data, CTC number was significantly higher in nude mice overexpressing MMP7/9, and the highest CTC count was observed when MMP7 and MMP9 were upregulated together. In contrast, MMP7 and MMP9 knockdown significantly decreased CTC number in explanted tumors.

\section{Combined evaluation of CTCs, MMP7, and MMP9 presents a more accurate clinical prediction of LADC metastasis status and prognosis}

Next, we applied ROC analysis, Kaplan-Meier analysis, and log-rank tests to estimate the efficacy of CTC count, serum MMP7 expression, and serum MMP9 expression as single and combined candidate prognostic biomarkers. As expected, ROC curve analysis revealed that the optimal cutoffs for CTC number, serum MMP7 level, and serum MMP9 level had great sensitivity and specificity for metastasis individually with an area under the curve (AUC) of $0.945(95 \%$ confidence interval (CI): 0.900-0.990; $p<0.01$; Figure 6A), 0.949 (95\% CI: 0.913-0.986; $p<0.01$; Figure 6B), and 0.930 (95\% CI: 0.881-0.979; $p<0.01$; Figure 6C), respectively. Notably, the combination cutoff, which had optimal sensitivity and specificity for metastasis with an AUC of
0.950 (95\% CI: 0.909-0.992; $p<0.01$; Figure 6D), showed a significant improvement.

According to the survival analysis, CTC count, serum MMP7 level, and serum MMP9 level exhibited favorable roles in predicting low survival rates individually (Figure 7A), with median survival rates of 1.83, 2.08, and 1.75 years (Figure 7B), respectively. Based on the univariate analysis, CTC count, serum MMP7 level, and serum MMP9 level were also significantly correlated with OS $(p<0.01$; Table 2). Compared with the tumors characterized by either overexpressing MMP7/MMP9 or having an elevated CTC count, those with an increased CTC count concomitant with MMP7 and MMP9 expression were associated with the poorest survival rate (median survival: 1.75 years; Figure 7B).

\section{Discussion}

Metastasis is the fatal determinant in various solid tumors and is often associated with poor prognosis. The early occurrence of micrometastasis sites in LADC makes it one of the most malignant tumors worldwide, which prompts us to identify reliable diagnostic indicators for early diagnosis of the disease in order to halt the progression of the disease timely. ${ }^{3}$ Since primary tumors constantly liberate CTCs into the circulation at all stages, CTC isolation and analysis have emerged as an exciting area of study as it can obtain serum tumor cells in a highly feasible, noninvasive, and dynamic manner. This would allow for the continuous surveillance 


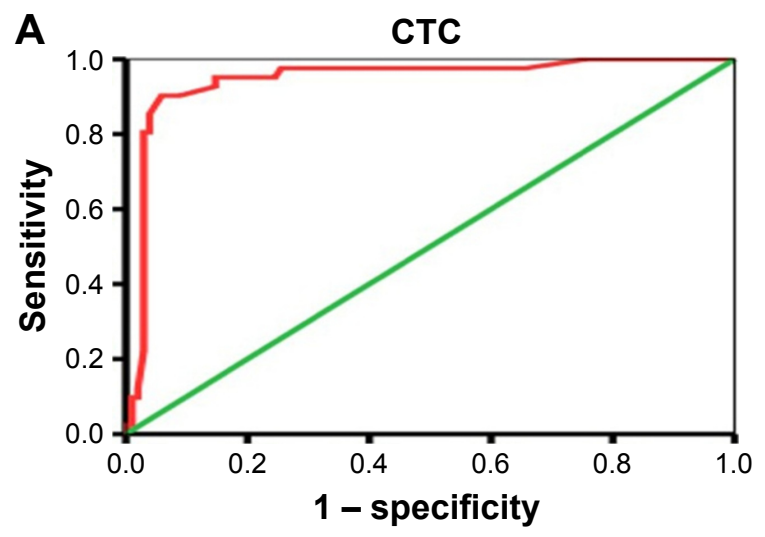

B

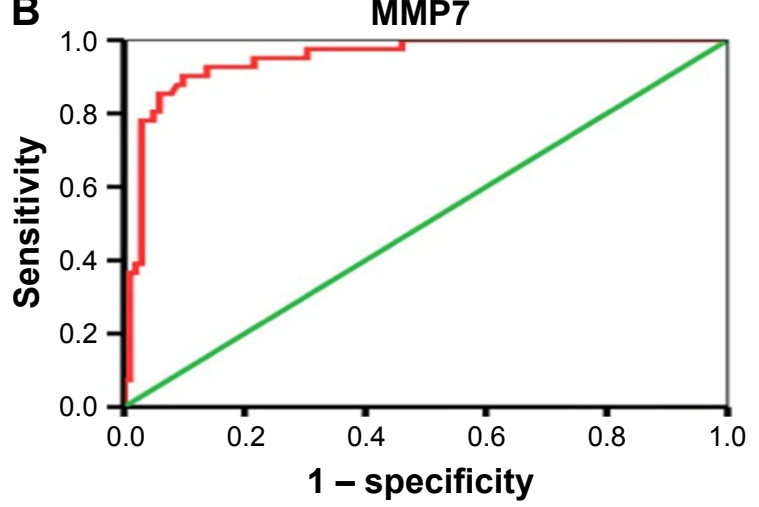

C

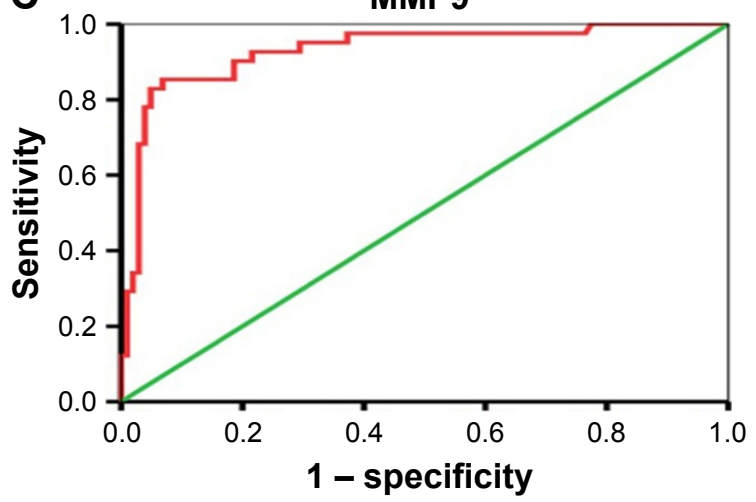

D

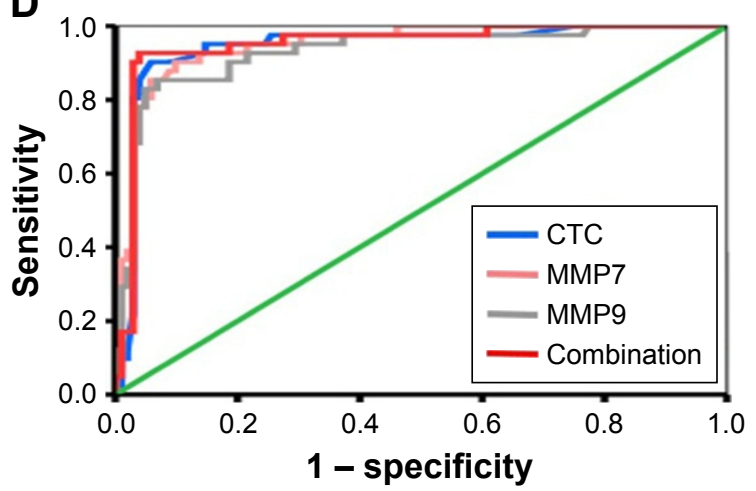

ROC analysis of CTC number

\begin{tabular}{ll}
\hline CTC number & Metastasis \\
\hline Sensitivity & 0.902 \\
Specificity & 0.941 \\
AUC & 0.945 \\
$95 \% \mathrm{Cl}$ & $0.900-0.990$ \\
\hline
\end{tabular}

ROC analysis of MMP7

\begin{tabular}{ll}
\hline MMP7 & Metastasis \\
\hline Sensitivity & 0.902 \\
Specificity & 0.902 \\
AUC & 0.949 \\
$95 \% \mathrm{Cl}$ & $0.913-0.986$ \\
\hline
\end{tabular}

ROC analysis of MMP9

\begin{tabular}{ll}
\hline MMP9 & Metastasis \\
\hline Sensitivity & 0.854 \\
Specificity & 0.931 \\
AUC & 0.930 \\
$95 \% \mathrm{Cl}$ & $0.881-0.979$ \\
\hline
\end{tabular}

ROC analysis of predicted probability

\begin{tabular}{ll}
\hline Predicted probability & Metastasis \\
\hline Sensitivity & 0.927 \\
Specificity & 0.961 \\
AUC & 0.950 \\
$95 \% \mathrm{Cl}$ & $0.909-0.992$ \\
\hline
\end{tabular}

Figure 6 Metastatic significance of concomitant CTC count, serum MMP7 expression, and serum MMP9 expression.

Notes: The cutoff values for CTC number, serum MMP7 level, and serum MMP9 level had great sensitivity and specificity for metastasis, with an area under the curve (AUC) of (A) 0.945 (95\% Cl: 0.900-0.990; $p<0.01$ ), (B) 0.949 (95\% Cl: 0.9I3-0.986; $p<0.0$ I), and (C) 0.930 ( $95 \%$ Cl: 0.88I-0.979; $p<0.01$ ), respectively. (D) The predicted cutoff value of the combination of CTC count, serum MMP7 level, and serum MMP9 level had an optimal AUC of 0.950 (95\% Cl: 0.909-0.992; $p<0.01$ ).

Abbreviations: CTC, circulating tumor cell; MMP7/9, matrix metalloproteinase $7 / 9$. 
A
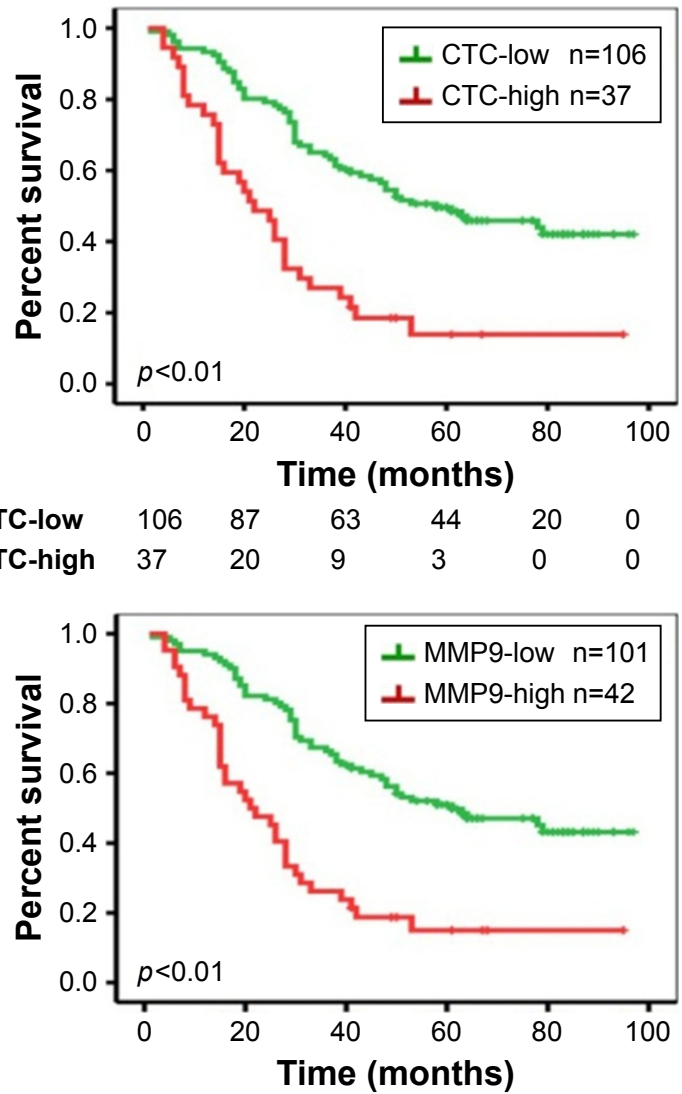

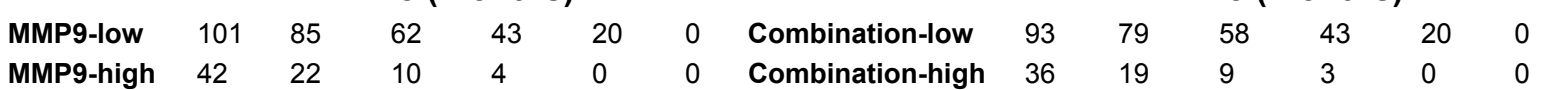

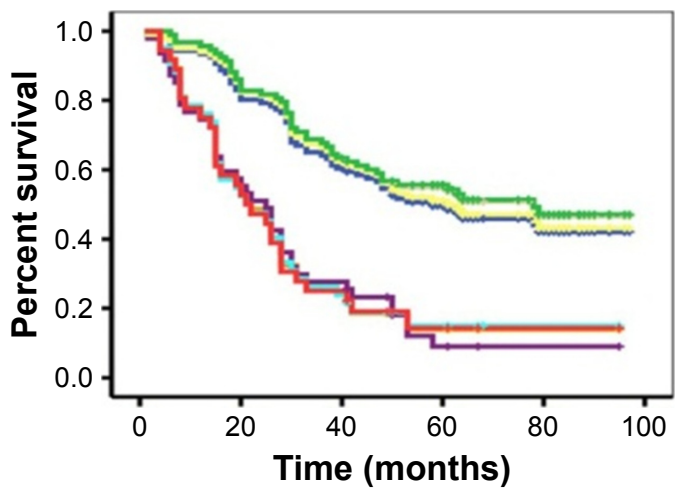

\begin{tabular}{|ll|}
\hline$\perp$ CTC-low & $\mathrm{n}=106$ \\
$\perp$ CTC-high & $\mathrm{n}=37$ \\
$\perp$ MMP7-low & $\mathrm{n}=96$ \\
$\perp$ MMP7-high & $\mathrm{n}=47$ \\
$\perp$ MMP9-low & $\mathrm{n}=101$ \\
$\perp$ MMP9-high & $\mathrm{n}=42$ \\
$\perp$ Combination-low & $\mathrm{n}=93$ \\
$\perp$ Combination-high & $\mathrm{n}=36$ \\
\hline
\end{tabular}

B

Overall survival (years)

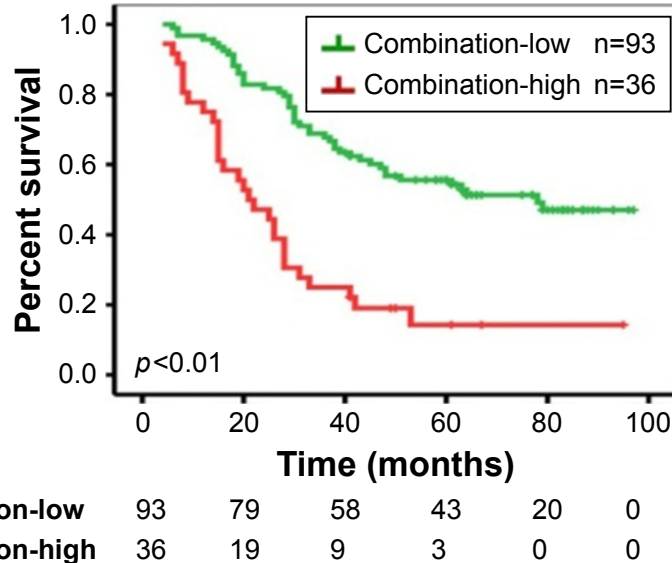

\begin{tabular}{|c|c|c|c|c|c|c|c|c|}
\hline & \multicolumn{2}{|c|}{ CTC number } & \multicolumn{2}{|c|}{ MMP7 } & \multicolumn{2}{|l|}{ MMP9 } & \multicolumn{2}{|c|}{ Combination } \\
\hline & High & Low & High & Low & High & Low & High & Low \\
\hline Median survival (year) & 1.83 & 4.83 & 2.08 & 6.50 & 1.75 & 5.08 & 1.75 & 6.50 \\
\hline 5-year survival rate & $13.9 \%$ & $49.6 \%$ & $9.0 \%$ & $55.0 \%$ & $15.0 \%$ & $51.0 \%$ & $14.3 \%$ & $55.6 \%$ \\
\hline
\end{tabular}

Figure 7 Increased CTC count, serum MMP7 level, and serum MMP9 level determine the worst prognosis.

Notes: (A) Kaplan-Meier curves revealed the shortest overall survival (OS). (B) The survival analysis revealed the shortest median survival and a lower 5 -year survival rate. Abbreviations: CTC, circulating tumor cell; MMP7/9, matrix metalloproteinase 7/9.

of cancer progression unlike traditional radiography and tumor biopsy ${ }^{6,28}$ However, the risk of losing tumor cells with features overlapping with blood cells and the low degree of accuracy limit the clinical utility of CTCs. To address this, the self-developed system NanoVelcro can reduce the number of missing EpCAM-negative CTCs by way of a microstructured surface that strengthens topographic interactions and a microfluidic chip that facilitates contact frequency according 
Table 2 Univariate and multivariate analyses of overall survival in LADC patients

\begin{tabular}{|c|c|c|c|c|c|c|}
\hline \multirow[t]{2}{*}{ Variable } & \multicolumn{3}{|c|}{ Univariate analysis } & \multicolumn{3}{|c|}{ Multivariate analysis } \\
\hline & Hazard ratio & $95 \% \mathrm{CI}$ & p-value & Hazard ratio & $95 \% \mathrm{Cl}$ & $p$-value \\
\hline Tumor size $(\mathrm{cm})$ & 1.314 & $0.835-2.066$ & 0.238 & 1.618 & $1.008-2.596$ & 0.046 \\
\hline Age & 2.754 & $|.879-4.92|$ & 0.138 & 2.467 & $1.004-3.895$ & 0.201 \\
\hline Gender & 1.974 & $1.117-4.72 \mid$ & 0.237 & 2.379 & $0.883-4.121$ & 0.109 \\
\hline Smoking & 3.513 & $2.135-5.938$ & $<0.001$ & 2.168 & $1.136-5.059$ & 0.014 \\
\hline Differentiation & 2.673 & $1.732-3.895$ & 0.133 & 1.934 & $0.635-5.128$ & 0.078 \\
\hline Metastasis & 3.488 & $2.252-5.402$ & $<0.001$ & 2.279 & $0.992-5.235$ & 0.052 \\
\hline Clinical stage & 3.719 & $2.147-6.443$ & $<0.001$ & 2.090 & $1.018-4.290$ & 0.044 \\
\hline CTC counts & 1.235 & $1.144-1.333$ & $<0.001$ & 0.287 & $0.086-0.959$ & 0.043 \\
\hline MMP7 expression & 3.308 & $2.155-5.076$ & $<0.001$ & 2.251 & $1.074-4.716$ & 0.032 \\
\hline MMP9 expression & 2.979 & $1.926-4.608$ & $<0.001$ & $\mathrm{I} .876$ & $0.64 I-5.495$ & 0.251 \\
\hline
\end{tabular}

Note: Statistical significance $(p<0.05)$ is shown in bold.

Abbreviations: CTC, circulating tumor cell; MMP7/9, matrix metalloproteinase 7/9; $\mathrm{Cl}$, confidence interval.

to our previous work. NanoVelcro has been shown to have an approximately 4-fold improved capture efficacy compared with CellSearch, and NanoVelcro appears to be a desirable alternative assay with high prognostic value. ${ }^{20}$ Here, we further applied NanoVelcro to quantify CTCs in a cohort of 143 patients to unveil the role of CTCs in the metastasis and progression of LADC.

Numerous prior reports have implicated the prognostic power of CTCs in SCLC, breast, colorectal, and prostate cancers. ${ }^{7-10}$ Our results first demonstrated that the CTC count captured by NanoVelcro was significantly higher in LADC patients with metastasis than in those without metastasis. Furthermore, a high level of significance between the number of CTCs and the pTNM stage was also observed, which further confirmed that CTC count can be treated as a valid biomarker of malignant progression in LADC. Moreover, our results revealed that no correlation existed between tumor burden and CTC count, which was in contrast to multiple reports that implicated a link between tumor burden and CTC count in NSCLC. ${ }^{29-31}$ We considered that the latent causes of the aforementioned paradoxical reports were that the included participants were all from two selected hospitals and the number of participants was limited, indicating that additional randomized controlled multicenter clinical studies in different populations and larger cohorts are required to investigate the intriguing association between CTCs and tumor size in LADC.

ECM proteins, which constitute a vital part of the basement membrane (BM), have been extensively studied in tumor invasion and metastasis. At the invasive fronts of primary tumors, the ECM is degraded by members of the MMP family of zinc-containing proteolytic enzymes. ${ }^{12,15,25}$ MMP7 and MMP9 were closely linked to tumor dissemination and poor clinical outcomes in NSCLC, breast, pancreatic, adrenocortical, and renal cancers. ${ }^{23-27}$ Here, we showed that upregulated expression of MMP7/9 predicts a higher risk of metastasis and a more advanced stage, and we revealed, for the first time, that CTC count is positively correlated with serum levels of MMP7 and MMP9 in LADC. Thus, we speculated whether the ectopic overexpression of MMP7/9 promotes CTCs to shed into the circulation by breaching the continuity of the BM or by digesting the ECM. Fukuda et $\mathrm{al}^{27}$ suggested that tumor cells in MMP7 knockout mice cannot be separated from the primary foci, thus showing a reduction of metastatic disease for patients with pancreatic ductal adenocarcinoma. Additionally, according to Cho et al, ${ }^{24}$ both transcripts and zymogens of MMP9 are closely linked to metastasis and other prognostic variables in renal cell carcinoma. Our results highlighted that abundant expression of MMP7 and MMP9 inhibited adhesion, enhanced metastasis, and increased CTC number, while suppression of MMP7 and MMP9 promoted adhesion, inhibited metastasis, and decreased serum CTCs. Both our in vitro and in vivo studies further confirmed that MMP7 and MMP9 are involved in the detachment of CTCs and that they may facilitate the following formation of regional and distant metastatic sites via the proteolysis of the ECM.

Serum MMP7 and MMP9 overexpression increase CTC numbers and present predictive potential for metastasis individually. Additionally, the results of our ROC analysis revealed that the combination of CTC count, serum MMP7 expression, and serum MMP9 expression has the highest sensitivity and specificity for metastasis. Furthermore, in our survival analysis, we compared the prognosis of patients with differences in our potential biomarkers. We found that the survival curves of combination-low and combinationhigh were the lowermost and uppermost curves, indicating the poorest prognosis and the longest OS, respectively. 
The aforementioned findings showed, for the first time, that a biomarker panel including elevated CTC number, MMP7 expression, and MMP9 expression predicts metastasis and prognosis in LADC. To date, no investigation has attempted to elucidate the underlying correlation among these biomarkers of poor prognosis. We proposed an invaluable panel for continuous monitoring of prognosis, optimized personal therapy, and investigation of metastatic mechanisms. Along with the clinical applicability of our predictive panel, which is composed of CTC, MMP7, and MMP9, we firmly believe that alternative treatment strategies can be modified in a timely manner and that the 5-year survival rate of LADC will be extended.

In summary, MMP7 and MMP9 are involved in the liberation of CTCs from primary tumors into the circulation. Moreover, CTC number, MMP7 expression, and MMP9 expression can represent a favorable biomarker panel to predict LADC metastasis and prognosis with improved sensitivity and specificity.

\section{Acknowledgments}

We thank Liantang Wang, Yuefeng Wang, Qiong He, Yongmei Cui, and Han Wang for the acquisition of data, and also Zhiqiang Cheng and Jianhong Wang for the related revision of this paper. This work was supported by grants from National Natural Science Foundation of China (30900650, 81372501, 81572260, 81172232, 81570008, 81172564, 81773299, and 31430030), Guangdong Natural Science Foundation (2011B031800025, S2012010008378, S2012010008270, S2013010015327, 2013B021800126, 20090171120070, 9451008901002146, 2013B021800126, 2014A030313052, 2014J4100132, 2015A020214010, 2016A020215055, and 2013B021800259), and the Guangzhou Science and Technology Planning Program (201704020094).

\section{Disclosure}

The authors report no conflicts of interest in this work.

\section{References}

1. Ferlay J, Soerjomataram I, Dikshit R, et al. Cancer incidence and mortality worldwide: Sources, methods and major patterns in GLOBOCAN 2012. Int $J$ Cancer. 2015;136(5):E359-E386.

2. Siegel RL, Miller KD, Jemal A. Cancer Statistics, 2017. CA Cancer JClin. 2017;67(1):7-30.

3. Consonni D, Pierobon M, Gail MH, et al. Lung cancer prognosis before and after recurrence in a population-based setting. $J$ Natl Cancer Inst. 2015;107(6):v59.

4. Passlick B, Izbicki JR, Kubuschok B, et al. Immunohistochemical assessment of individual tumor cells in lymph nodes of patients with non-small-cell lung cancer. J Clin Oncol. 1994;12(9):1827-1832.

5. Herbst RS, Heymach JV, Lippman SM. Lung cancer. $N$ Engl J Med. 2008;359(13):1367-1380.
6. Lianidou ES, Strati A, Markou A. Circulating tumor cells as promising novel biomarkers in solid cancers. Crit Rev Clin Lab Sci. 2014; 51(3):160-171.

7. Budd GT, Cristofanilli M, Ellis MJ, et al. Circulating tumor cells versus imaging - predicting overall survival in metastatic breast cancer. Clin Cancer Res. 2006;12(21):6403-6409.

8. Cohen SJ, Punt CJ, Iannotti N, et al. Prognostic significance of circulating tumor cells in patients with metastatic colorectal cancer. Ann Oncol. 2009;20(7):1223-1229.

9. de Bono JS, Scher HI, Montgomery RB, et al. Circulating tumor cells predict survival benefit from treatment in metastatic castration-resistant prostate cancer. Clin Cancer Res. 2008;14(19):6302-6309.

10. Salgia R, Weaver RW, McCleod M, et al. Prognostic and predictive value of circulating tumor cells and CXCR4 expression as biomarkers for a CXCR4 peptide antagonist in combination with carboplatinetoposide in small cell lung cancer: exploratory analysis of a phase II study. Invest New Drug. 2017;35(3):334-344.

11. Hamilton G, Rath B, Klameth L, Hochmair MJ. Small cell lung cancer: Recruitment of macrophages by circulating tumor cells. Oncoimmunology. 2016;5(3):e1093277.

12. Bonnans $\mathrm{C}$, Chou J, Werb Z. Remodelling the extracellular matrix in development and disease. Nat Rev Mol Cell Biol. 2014;15(12):786-801.

13. Castro-Castro A, Marchesin V, Monteiro P, Lodillinsky C, Rosse C, Chavrier P. Cellular and molecular mechanisms of MT1-MMPdependent cancer cell invasion. Annu Rev Cell Dev Biol. 2016;32: 555-576.

14. Chevalier C, Collin G, Descamps S, et al. TOM1L1 drives membrane delivery of MT1-MMP to promote ERBB2-induced breast cancer cell invasion. Nat Commun. 2016;7:10765.

15. Egeblad M, Werb Z. New functions for the matrix metalloproteinases in cancer progression. Nat Rev Cancer. 2002;2(3):161-174.

16. Page-McCaw A, Ewald AJ, Werb Z. Matrix metalloproteinases and the regulation of tissue remodelling. Nat Rev Mol Cell Biol. 2007;8(3): 221-233.

17. Zhang J, Luo J, Ni J, et al. MMP-7 is upregulated by COX-2 and promotes proliferation and invasion of lung adenocarcinoma cells. Eur $J$ Histochem. 2014;58(1):2262.

18. Cox G, Jones JL, O'Byrne KJ. Matrix metalloproteinase 9 and the epidermal growth factor signal pathway in operable non-small cell lung cancer. Clin Cancer Res. 2000;6(6):2349-2355.

19. Sun S, Ke Z, Wang F, et al. Overexpression of astrocyte-elevated gene-1 is closely correlated with poor prognosis in human non-small cell lung cancer and mediates its metastasis through up-regulation of matrix metalloproteinase-9 expression. Hum Pathol. 2012;43(7):1051-1060.

20. He W, Xu D, Wang Z, et al. Detecting ALK-rearrangement of CTC enriched by nanovelcro chip in advanced NSCLC patients. Oncotarget. Epub 2016 Mar 23.

21. Pan Y, Guo X, Yang Z, Chen S, Lei Y. AEG-1 activates Wnt/PCP signaling to promote metastasis in tongue squamous cell carcinoma. Oncotarget. 2016;7(2):2093-2104.

22. Koshiba T, Hosotani R, Miyamoto Y, et al. Expression of stromal cell-derived factor 1 and CXCR4 ligand receptor system in pancreatic cancer: a possible role for tumor progression. Clin Cancer Res. 2000; 6(9):3530-3535.

23. Pinzani P, Scatena C, Salvianti F, et al. Detection of circulating tumor cells in patients with adrenocortical carcinoma: a monocentric preliminary study. J Clin Endocrinol Metab. 2013;98(9):3731-3738.

24. Cho NH, Shim HS, Rha SY, et al. Increased expression of matrix metalloproteinase 9 correlates with poor prognostic variables in renal cell carcinoma. Eur Urol. 2003;44(5):560-566.

25. Liu D, Nakano J, Ishikawa S, et al. Overexpression of matrix metalloproteinase-7 (MMP-7) correlates with tumor proliferation, and a poor prognosis in non-small cell lung cancer. Lung Cancer. 2007; 58(3):384-391.

26. Jacob A, Jing J, Lee J, et al. Rab40b regulates trafficking of MMP2 and MMP9 during invadopodia formation and invasion of breast cancer cells. J Cell Sci. 2013;126(Pt 20):4647-4658. 
27. Fukuda A, Wang SC, Morris JT, et al. Stat3 and MMP7 contribute to pancreatic ductal adenocarcinoma initiation and progression. Cancer Cell. 2011;19(4):441-455.

28. Plaks V, Koopman CD, Werb Z. Cancer. Circulating tumor cells. Science. 2013;341(6151):1186-1188.

29. Chen X, Wang X, He H, Liu Z, Hu JF, Li W. Combination of circulating tumor cells with serum carcinoembryonic antigen enhances clinical prediction of non-small cell lung cancer. PLoS One. 2015; 10(5):e126276.
30. Hofman V, Bonnetaud C, Ilie MI, et al. Preoperative circulating tumor cell detection using the isolation by size of epithelial tumor cell method for patients with lung cancer is a new prognostic biomarker. Clin Cancer Res. 2011;17(4):827-835.

31. Zhang Z, Xiao Y, Zhao J, et al. Relationship between circulating tumour cell count and prognosis following chemotherapy in patients with advanced non-small-cell lung cancer. Respirology. 2016;21(3): 519-525. 


\section{Supplementary materials}

Table SI siRNA sequences used in this study

\begin{tabular}{ll}
\hline Gene & Sequences $\left(5^{\prime} \mathbf{3}^{\prime}\right)$ \\
\hline MMP7 & GACCTTATGGCTACAGTAA \\
MMP9 & GCTGCAGTTTGATGATGAA
\end{tabular}

Table S2 PCR primers used in this study

\begin{tabular}{lll}
\hline Gene & Forward primer & Reverse primer \\
\hline MMP7 & ATGCGACTCACCGTG & CTATTTCTTTCTTGAATTACTT \\
MMP9 & ATGAGCCTCTGGCAGCCCCT & CTAGTCCTCAGGGCACTGCAGG
\end{tabular}

Abbreviations: MMP7/9, matrix metalloproteinase 7/9; PCR, polymerase chain reaction.

\section{Publish your work in this journal}

The International Journal of Nanomedicine is an international, peerreviewed journal focusing on the application of nanotechnology in diagnostics, therapeutics, and drug delivery systems throughout the biomedical field. This journal is indexed on PubMed Central, MedLine, CAS, SciSearch ${ }^{\circledR}$, Current Contents ${ }^{\circledR} /$ Clinical Medicine,
Journal Citation Reports/Science Edition, EMBase, Scopus and the Elsevier Bibliographic databases. The manuscript management system is completely online and includes a very quick and fair peer-review system, which is all easy to use. Visit http://www.dovepress.com/ testimonials.php to read real quotes from published authors.

Submit your manuscript here: http://www.dovepress.com/international-journal-of-nanomedicine-journal 